\title{
REPRESENTACIÓN DEL "SCORE” GLEASON EN LA BIOPSIA PROSTÁTICA DEL "SCORE" GLEASON DEFINITIVO DE LA PROSTATECTOMÍA RADICAL
}

\author{
F. ALGABA ARREA*,**, A. CHIVITE DE LEÓN*, J.Mª SANTAULARIA SEGURA*, \\ A. OLIVER SAMPER***

\begin{abstract}
*Sección de Patología de la Fundación Puigvert. Barcelona.
**Departamento de Ciencias Morfológicas de la Facultad de Medicina. Universidad Autónoma de Barcelona. ***Servicio de Laboratorio Central de la Fundación Puigvert. Barcelona.
\end{abstract}

Actas Urol Esp. 28 (1): 21-26, 2004

\section{RESUMEN}

REPRESENTACIÓN DEL "SCORE" GLEASON EN LA BIOPSIA PROSTÁTICA DEL "SCORE" GLEASON DEFINITIVO DE LA PROSTATECTOMÍA RADICAL

INTRODUCCIÓN: El grado de diferenciación del adenocarcinoma de próstata por el método de Gleason ("score" Gleason) (sG) es un factor pronóstico post-prostatectomía radical, por lo que su representatividad en la biopsia es de sumo interés.

PACIENTES Y MÉTODOS: Doscientos quince pacientes con determinación del sG en la biopsia y la pieza de prostatectomía, determinado a ciegas por el mismo equipo de tres patólogos.

RESULTADOS: Coincidencia exacta en un 49,7\%, infravaloración en la biopsia en un 38,6\% y sobrevaloración en la biopsia en un $11,6 \%$.

El análisis por sG revela: ausencia de casos con sG 2 en la biopsia. No-reproducibilidad en la pieza de los sG 3 y 4 de las biopsias. Coincidencia de los sG 5, 6, 7, 8 y 9 en biopsia del 25\%, 45\%, 72,7\%, 36,8\% y 60\%, respectivamente ( $\kappa$ global 0,32 $\pm 0,047$, $\mathrm{p}<0,0001$ en sG de 5 a 8).

El patrón 4 de Gleason es el menos reconocido o representado en las biopsias ( $40 \%$ de los que se encuentran en la prostatectomía no han sido detectados en la biopsia).

CONCLUSIONES: La determinación del grado en la biopsia por el método de Gleason tiene una buena correlación con el de la pieza de prostatectomía. Para mejorar la correspondencia se deben tener en cuenta que los sG 2, 3 y 4 en biopsia no son reproducidos en las prostatectomías y el porcentaje de coincidencias aumenta con el sG, siendo los patrones 4 y 5 los menos reconocidos en la biopsia, por lo que se recomienda cautela en casos de patrones bajos, en especial con escasa representación de tumor, y una escrupulosa búsqueda de los patrones 4 y 5 y reflejarlos, por escasos que sean en la biopsia.

PALABRAS CLAVE: Patologia cáncer próstata. Sistema Gleason. Biopsia próstata. Prostatectomia radical.

\section{ABSTRACT}

EVIDENCE OF THE RADICAL PROSTATECTOMY GLEASON SCORE IN THE BIOPSY GLEASON SCORE

INTRODUCTION: The Gleason score (Gs) for prostatic cancer has a good prognosis correlation after radical prostatectomy, for this reason its correlation with the Gs in the biopsy can be useful.

PATIENTS AND METHODS: Two hundred fifteen patients with blind evaluation among three pathologists of their Gs in biopsy and in the corresponding radical prostatectomy specimen are presented.

RESULTS: The exact coincidence is present in $49.7 \%$ of cases, $38.6 \%$ of cases are under graded in the biopsy and $11.6 \%$ of them over graded in the biopsy.

No cases of Gs 2 in the biopsy are found. Any case with Gs 3 and 4 in the biopsy are reproduced in the radical prostatectomy specimen. The exact coincidence for biopsy Gs 5, 6, 7, 8 and 9 are 25\%, 45\%, 72.7\%, 36.6\% and 60\% respectively ( $\mathrm{\kappa} 0.32 \pm 0.047$, $\mathrm{p}<0.0001$ in Gs 5 to 8).

The Gleason pattern 4 is the less diagnosed in prostate biopsies (40\% of cases with this pattern in the excision specimen it is missing in the biopsy).

CONCLUSIONS: The Gs in the needle prostatic biopsy has a good correspondence with the Gs in the radical prostatectomy specimen. For an increase of the reproducibility it is recommendable avoid the diagnosis of Gs 2,3 and 4 in biopsy and a scrupulous search for the patterns 4 and 5 .

KEY WORDS: Prostatic cancer pathology. Gleason system. Needle prostatic biopsy. Radical prostatectomy. 
$\mathrm{E}_{\mathrm{B}}^{1}$ pronóstico de la evolución de un paciente prostatectomizado por adenocarcinoma depende de diversos factores, entre los que el nivel sérico del antígeno prostático específico ${ }^{1}$, el confinamiento o no del carcinoma al órgano, el estado de los márgenes quirúrgicos y el grado de diferenciación ("score" Gleason) (sG) en la pieza, son los más certeros ${ }^{2,3}$. A parte del antígeno prostático específico sérico, sólo el grado de diferenciación de las biopsias prostáticas puede ser conocido antes de la prostatectomía y por ello el saber la fiabilidad de la representación del sG final y cuáles son las causas de las eventuales discordancias, es de sumo interés práctico y objeto del presente trabajo.

\section{PACIENTES Y MÉTODOS}

Se han estudiado 215 pacientes consecutivos de los que se dispone de las biopsias transrectales diagnósticas (con un mínimo de seis cilindros, tres por lado, realizadas con aguja de 18 gauge) y la pieza de prostatectomía radical incluida en su totalidad con cortes completos panorámicos. En ninguno de estos pacientes nos consta que haya recibido tratamiento entre la biopsia y la cirugía radical.

La evaluación de los grados es el resultado del trabajo cotidiano de tres patólogos. El diagnóstico de cada muestra (sea la biopsia o próstata completa) ha sido hecho por un solo patólogo y las piezas de prostatectomía han sido evaluadas por el patólogo que le ha correspondido el caso, hubiera visto o no, la biopsia previa, sin prever la biopsia. Las preparaciones no han sido revisadas de nuevo para esta publicación. Con todo ello, se intenta exponer la experiencia del diagnóstico habitual en nuestra sección de patología.

El grado de diferenciación se ha determinado según los criterios de Gleason ${ }^{4}$, usando sólo el patrón primario y el secundario. En la biopsia se ha considerado como representativo el Gleason global $^{5}$. Se ha contabilizado el número de cilindros positivos y el porcentaje que representa, en cada paciente, con respecto al número total de cilindros que se le han extraído.

Análisis estadístico: los resultados se han expresado en porcentajes e intervalo de confianza del 95\%. La comparación de proporciones se ha efectuado mediante tablas de contingencia y test del $\chi 2$. Se ha utilizado la kappa de Cohen para analizar la concordancia entre el sG de la biopsia y la pieza de prostatectomía radical. Las variaciones del porcentaje de cilindros positivos en la biopsia según el sG se han analizado mediante una ANOVA. Se considera significativa una $\mathrm{p} \leq 0,05$.

\section{RESULTADOS}

En 107 pacientes (49,7\%) el sG de la biopsia y el de la prostatectomía ha coincidido, en 83 enfermos $(38,6 \%)$ se ha infravalorado el sG en la biopsia con respecto al de la prostatectomía, y en $25(11,6 \%)$ se ha emitido un sG en la biopsia superior al de la prostatectomía.

Si analizamos la coincidencia por cada $\mathrm{SG}$ diagnosticado en las biopsias (Fig. 1) se constata que no ha habido ningún caso diagnosticado de sG 2 en la biopsia, 7 pacientes se han valorado como sG 3 ó 4 en las biopsias, y ninguno de ellos se ha reproducido en la pieza de prostatectomía, y sólo 9 de los 36 (25\%) diagnosticados como sG 5 se ha encontrado éste en la pieza de exéresis. Los sG 6 y 7 han sido los más reproducidos en las piezas de prostatectomía, con un $45 \%$ y un $72,7 \%$ de exacta coincidencia, respectivamente. Los casos con sG 8 en la biopsia tienen un 36,8\% de coincidencia y los sG 9 del 60\%; con lo que la $\kappa$ global para los sG 5 a 8 es de 0,32 $\pm 0,047$ (p<0,0001). Al agrupar las biopsias como sG $<5$ ó $>5$, el 96,5\% muestran una concordancia cuando es $\mathrm{sG}>5$ y un $27,9 \%$ cuando es $\mathrm{sG}<5$, lo que representa una $\kappa=0,312 \pm 0,082(\mathrm{p}<0,0001)$. Esta concordancia es superior $(\kappa=0,35 \pm 0,056)$ al agrupar los casos en sG de la biopsia en <6 ó >6.

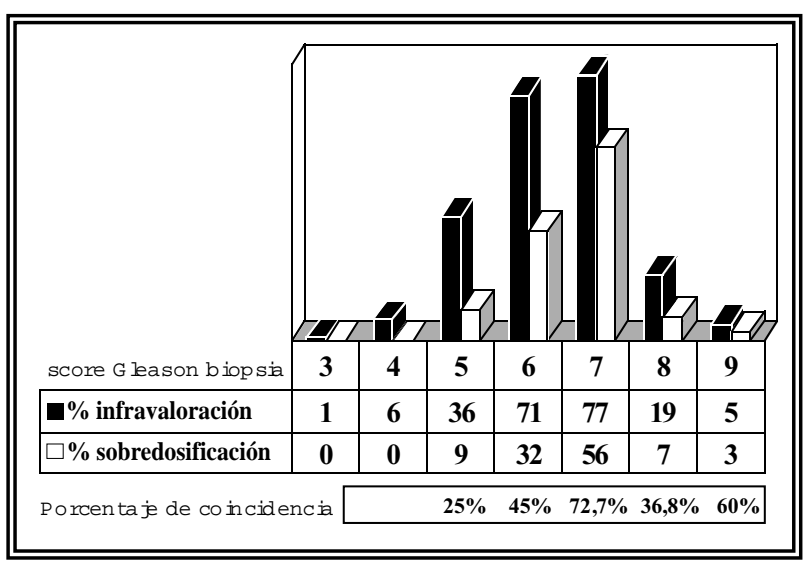

FIGURA 1. Coincidencia entre el "score" Gleason de la biopsia y el de la prostatectomia radical. 
Los porcentajes de infravaloraciones del sG de la biopsia con respecto al de la prostatectomía (Fig. 2) demuestran mayor infravaloración en los sG $5,72,2 \%$ (del 55 al 86\%) y sG 6, 49\% (del 37 al 61\%); mientras que los sG 7 y 8 sólo son superados en la pieza de prostatectomía en un $16,8 \%$ (del 9,3 al $27,1 \%$ ) y un $10,5 \%$ (del 1,3 al $33,1 \%$ ), respectivamente.

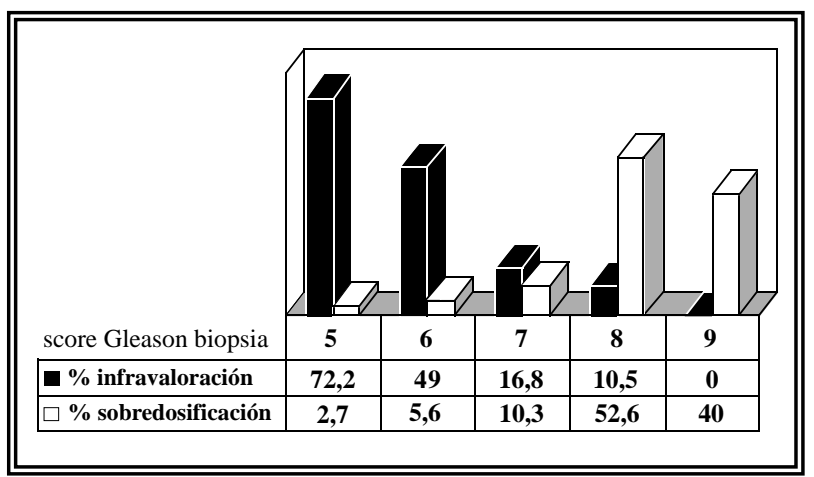

FIGURA 2. Infravaloración y sobrevaloración de la biop sia con respecto a la prostatectomia radical.

Aunque mucho menos frecuente también hay sobrevaloraciones en la biopsia con respecto al sG de la pieza de prostatectomía, especialmente en el sG 8, 52,6\% (del 29 al 76\%) y el sG 9, 40\% (del 5 al 85\%).

$\mathrm{Al}$ evaluar el origen de la discrepancia entre el sG de la biopsia y el de la pieza de prostatectomía se constata que en 20 enfermos con sG 5 en la biopsia, éste es 7 en la prostatectomía $(76,9 \%$ de los infravalorados), y 32 pacientes con sG 6 en la biopsia, éste es 7 en la prostatectomía $(94,3 \%$ de los infravalorados), consecuencia de un patrón Gleason 4 no representado o no reconocido en la biopsia.

$\mathrm{Si}$ cuantificamos los patrones Gleason no reproducidos vemos que en un $12,4 \%$ de enfermos se ha visto un patrón 3 en la biopsia que no se ha reproducido en la prostatectomía, y en un $8,9 \%$ el patrón 3 se ha visto en la prostatectomía no habiendo sido visto en la biopsia. Si hacemos esta misma valoración para el patrón 4, un 7,9\% de casos tienen este patrón en la biopsia, pero no en la prostatectomía, mientras que en un $40 \%$ de casos sólo se encuentra el patrón 4 en la pieza de prostatectomía.
Al estudiar la variación del porcentaje de cilindros positivos se observa que aumenta significativamente con el sG de la biopsia y por lo tanto también se relaciona con el incremento de coincidencia entre el sG de la biopsia y el de la pieza de prostatectomía (Fig. 3).

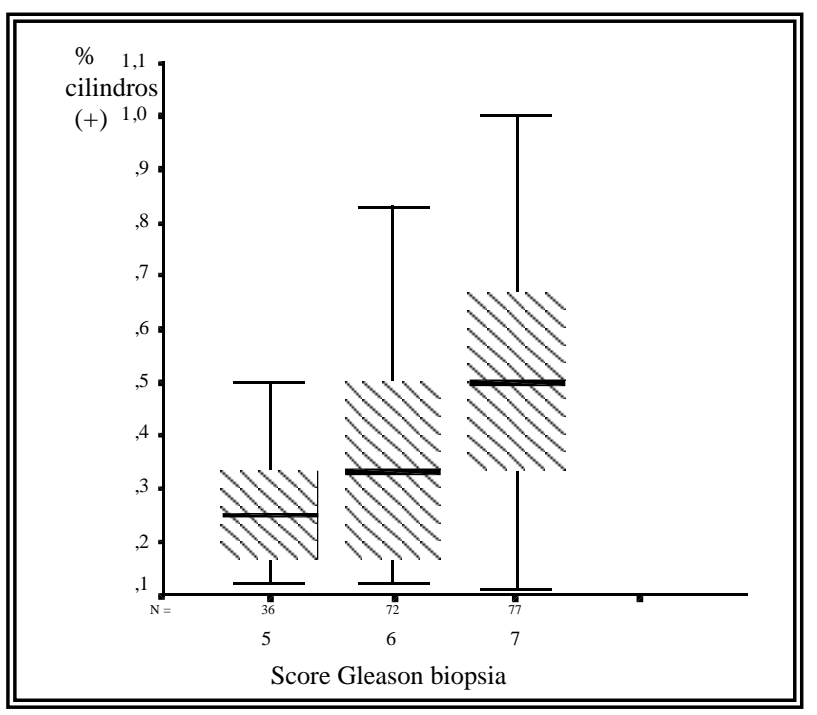

FIGURA 3. Porcentaje de cilindros positivos según score 5 , 6 y 7 de la biopsia.

\section{DISCUSIÓN}

Los hallazgos de esta serie son semejantes a los de otras publicaciones de la literatura ${ }^{6}$, con una incidencia de la infravaloración del sG de la biopsia algo más baja que en ellas $(p=0,09)$ (Tabla I), que a pesar de no ser estadísticamente significativa muestra una tendencia.

$\mathrm{El}$ análisis conjunto de la presente serie y otras de la literatura ${ }^{5-8}$ nos permite hacer varias consideraciones:

- Los sG 2, 3 y 4 en la biopsia no se reproducen en las piezas de prostatectomía, lo que ha comportado que hayan aparecido recomendaciones de no hacer su diagnóstico ya que pueden inducir una falsa sensación de baja agresividad ${ }^{7}$.

- La coincidencia de los SG es mayor contra más alto es éste en la biopsia (25\% en el 5, 45\% en el 6 y 72,7\% en el 7) destacando que en los sG 8 y 9 esta reproducibilidad disminuye, posiblemente por no ser capaces de reconocer mínimos focos de patrón 5 en la biopsia, aunque el escaso número de casos de estos $\mathrm{sG}$ en todas las series puede alterar los resultados. 


\section{TABLA I}

CORRESPONDENCIA ENTRE EL "SCORE" GLEASON DE LA BIOPSIA Y EL DE LA PROSTATECTOMÍA (COMPARÁNDOLA CON LA COMBINACIÓN DE VARIAS SERIES DE LA LITERATURA) ${ }^{6}$

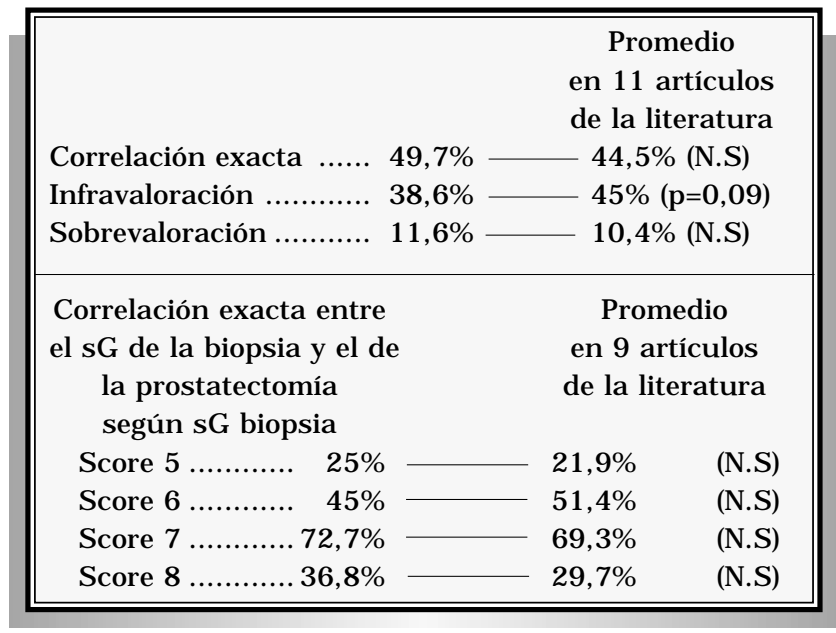

- El progresivo incremento de correlación entre el sG de la biopsia y el de la prostatectomía coincide con un mayor porcentaje de cilindros afectados por el adenocarcinoma, como si el volumen tumoral y el sG se relacionaran. Esta es una cuestión discutida en la literatura, y para algunos autores no hay relación entre el sG y el volumen tumoral ${ }^{6}$, por lo que aunque nosotros encontramos cierta relación volumen/sG, creemos que hay que seguir siendo precavido en los casos con bajos SG y escasa representación del carcinoma en la biopsia, porque puede disminuir su representatividad ${ }^{8}$.

- Si analizamos los errores por patrones vemos: a) que el patrón 3 de Gleason no se ve en la pieza de prostatectomía, en el 12,4\% de los casos en los que ha sido identificado en la biopsia, y en un $8,9 \%$ de los casos que es reconocido en la pieza de prostatectomía no ha sido visto en la biopsia, lo que puede ser expresión de un problema de interpretación o de muestreo; b) el patrón 4 no se ve en la prostatectomía en un $7,9 \%$ de casos vistos en la biopsia, (posiblemente por error de interpretación), pero llegan a un $40 \%$ los casos que muestran este patrón en la prostatectomía sin haberse visto en la biopsia, probablemente por un problema de muestreo, sin descartar una posible mala interpretación.

Ante estos resultados nos preguntamos cómo evitar los errores de interpretación. Para tratar de encontrar alguna respuesta hemos de reconside- rar los criterios para establecer los patrones de Gleason y las posibles fuentes de los errores de interpretación.

La guía principal para determinar los patrones de Gleason es la interrelación entre el tumor y el tejido de alrededor, esto no es posible de determinar en las biopsias, por lo que se usa una estrategia indirecta que consiste en evaluar la forma de las glándulas y la separación entre ellas, por ello pueden confundirse el patrón 1 (glándulas adosadas entre sí, pero con una mínima separación estromal entre ellas) y el patrón 4 (glándulas adosadas entre sí, sin estroma, y por lo tanto fusionadas) ${ }^{9}$; así pues en las biopsias debe temerse un error de interpretación en el caso de que veamos un patrón 1 , no sólo por lo comentado, sino también por lo infrecuente que es, ya que incluso en la descripción inicial sólo representó un 3,5\% de los casos y tan sólo se refiere un sG 2 (sumatorio del patrón 1 en caso de no haber patrón secundario) en menos del $1 \% 4$. Otra fuente de error lo constituye el patrón criboso, ya que si está bien delimitado es un patrón 3B, pero si los bordes son difuminados o imprecisos, es un patrón 4, llegando a ser un patrón 5 si se reconoce necrosis central, que no debe confundirse con el material proteináceo de la luz ${ }^{9}$.

Aparte de la interpretación de los patrones también puede inducir a error la multiplicidad de patrones coincidentes. Alrededor de un $4 \%$ de las biopsias tienen tres patrones de Gleason ${ }^{10}$ y un $3 \%$ llegan a tener cuatro patrones ${ }^{11}$. Este hecho justifica discordancias entre observadores, al variar los criterios sobre qué patrones tomar en consideración. La tendencia actual es la de incluir en la determinación del "score" global los patrones 4 y $5^{12}$, por escasos que sean en la biopsia ya que parecen tener implicaciones biológicas incluso en mínimas proporciones ${ }^{13}$.

Independientemente de una mayor precisión en el uso de los criterios de evaluación se han ido reconociendo aspectos de la gradación de Gleason con mejor correlación biológica, y así se están considerando como patrón 4 los adenocarcinomas mucosos coloides y como patrón 5 los carcinomas en anillo de sello, los de tipo linfoepiteliode y los sarcomatoides ${ }^{14}$, asimismo se indica en los sG 7 si el patrón principal es el $4^{15}$, y se recomienda cuantificar el porcentaje del patrón 4 y 5 . 
A pesar de todo la interpretación del patólogo sigue siendo uno de los factores más importantes para que el sG de la biopsia sea lo más semejante posible al sG de la pieza de prostatectomía radical y como es lógico la reproducibilidad entre los patólogos especializados es más alta que entre los patólogos generales ${ }^{16,17}$, pero está demostrado que la intercomunicación de los patólogos así como la publicación de textos, iconografía y programas de difusión de los patrones de Gleason conducen a una mayor reproducibilidad entre todos los patólogos sean o no especialistas ${ }^{18}$, y ello explica que en la literatura actual sean mayores las concordan$\operatorname{cias}^{19}$, que en la literatura de hace algunos años ${ }^{20}$.

En conclusión, vemos que la valoración del sG en la biopsia prostática tiene una buena correlación con el sG en la prostatectomía, no pudiéndose esperar una reproducibilidad exacta (que por otra parte no existe en ninguna actividad humana). Para mejorar la correspondencia entre la biopsia y la prostatectomía es recomendable una estricta observancia de los criterios, el dudar de los casos con un bajo sG, o de asociaciones de patrones de Gleason extremos (bien por mala interpretación o por insuficiente representatividad) y el tener en cuenta los patrones 4 y 5 por escasos que sean ya que la valoración del sG ha de ser más cualitativa que cuantitativa. Con estas guías y la progresiva adquisición de experiencia con las técnicas educativas más actuales de las que se dispone ${ }^{21}$, el sistema de gradación de Gleason es un buen método para el uso general en todos los centros y debe hacerse en las biopsias, ya que puede ayudar a la toma de decisiones terapéuticas ${ }^{3,22}$.

Agradecimientos: Agradecemos al Sr. J.A. Muñoz y a la Dra. L. López, del Servicio de Documentación Médica de la Fundación Puigvert, su ayuda en la gestión de los datos.

\section{REFERENCIAS}

1. KATTAN MW, WHEELER TM, SCARDINO PT. Postoperative nomogram for disease recurrence after radical prostatectomy for prostate cancer. $J$ Clin Oncol 1999; 17: 1499-1507.

2. EPSTEIN JI, PARTIN AW, SAUVAGEOT J, WALSH PC.: Prediction of progression following radical prostatectomy. A multivariate analysis of 721 men with long-term follow-up. Am J Surg Pathol 1996; 20: 286-292.
3. VILLAVICENCIO H, MILLÁN F, CHECHILE G, SALINAS D, VICENTE J.: Factores pronósticos y tablas predictivas del cáncer de próstata no localizado que excluirían la realización de la prostatectomía radical. Actas Urol Esp 1998; 22: 581-589.

4. GLEASON DF.: Histologic grading and clinical staging of carcinoma of the prostate. En Urologic Pathology. Editor Tannenbaum M. Editorial Lea \& Febiger 1977: 171-198. Filadelfia.

5. DUNN RL, SHAH R, ZHOU M, INGOLD C, RUBIN MA.: Global Gleason score, highest core Gleason score or weighted Gleason score. What Gleason score should be reported in prostate needle biopsy? Mod Pathol 2002; 5: 161 A (abstract).

6. CARLSON GD, CALVANESE CB, KAHANE H, EPSTEIN JI.: Accuracy of biopsy Gleason scores from a large uropathology laboratory: use of a diagnostic protocol to minimize variability. Urology 1998; 51: 525-529.

7. EPSTEIN JI.: Gleason score 2-4 adenocarcinoma of the prostate on needle biopsy. A diagnosis that should not be made. Am J Surg Pathol 2000; 24: 477-478.

8. RUBIN MA, DUNN R, KAMBHAM N, PEARSALL C, O־TOOLE KM.: Should a Gleason score be assigned to a minute focus of carcinoma on prostate biopsy? Am J Surg Pathol 2000; 24: 1634-1640.

9. ALLSBROOK WC, MANGOLD KA, YANG X, EPSTEIN JI.: The Gleason grading system. An overview. J Urol Pathol 1999; 10: 141-157.

10. MCGOWAN DG, BAIN GO, HANSON J.: Evaluation of histological grading (Gleason) in carcinoma of the prostate: adverse influence of highest grade. Prostate 1983; 4: 111-118.

11. RUIJTER ET, VAN DE KAA CA, SCHALKEN JA, DEBRUYNE FM, RUITER DJ.: Histological grade heterogeneity in multifocal prostate cancer. Biological and clinical implications. J Pathol 1996; 180: 295-299.

12. SRIGLEY JR, AMIN MB, BOSTWICK DG, GRIGNON DJ, HAMMOND EH.: Updated protocol for the examination of specimens from patients with carcinomas of the prostate gland. Arch Pathol Lab Med 2000; 124: 1034-1039.

13. PAN CC, POTTER SR, PARTIN AW, EPSTEIN JI.: The prognostic significance of tertiary Gleason patterns of higher grade in radical prostatectomy specimens: a proposal to modify the Gleason grading system. Am J Surg Pathol 2000; 24: 563-569.

14. BOSTWICK DG.: Pathology of prostate cancer. En Urologic Cancer. Editores Erntoff MS, Heaney JA, Peschel RE. Editorial Blackwell Science. Cambridge Massachusetts, 1997: 15-47.

15. MAKAROV DV, SANDERSON H, PARTIN AW, EPSTEIN JI.: Gleason score 7 prostate cancer on needle biopsy: is the prognostic different in Gleason scores $4+3$ and $3+4$ independent of the number of involved cores?. J Urol 2002; 167: 2440-2442.

16. ALLSBROOK WC, MANGOLD KA, JOHNSON MH, LANE RB, LANE CG, AMIN MB, BOSTWICK DG, HUMPHREY PA, JONES EC, REUTER VE, SARK W, SESTERHENN IA, TRONCOSO P, WHEELER TM, EPSTEIN JI.: Interobserver reproducibility of Gleason grading of prostatic carcinoma: urologic pathologists. Hum Pathol 2001; 32: 74-80. 
17. ALLSBROOK WC, MANGOLD KA, JOHNSON MH, LANE RB, LANE CG, EPSTEIN JI.: Interobserver reproducibility of Gleason grading of prostatic carcinoma: general pathologists. Hum Pathol 2001; 32: 81-88.

18. MIKAMI Y, MANABE T, EPSTEIN JI, SHIRAISHI T, FURUSATO M, TSUZUKI M, MATSUNO Y, SASANO H.: Accuracy of Gleason grading by practicing pathologists and the impact of education on improving agreement. Hum Pathol 2003; 34: 658-665.

19. GROSSFELD GD, CHANG JJ, BROERING JM, LI YP, LUBECK DP, FLANDERS. S, CARROLL PR.: Under staging and under grading in a contemporary series of patients undergoing radical prostatectomy: results from the cancer of the prostate strategic urologic research endevon database. $J$ Urol 2001; 165: 851-856.

20. COOKSON MS, FLESHNER NE, SOLOWAY SM, FAIR WR.: Correlation between Gleason score of needle biopsy and radical prostatectomy specimen: accuracy and clinical implications. J Urol 1997; 157: 559-562.
21. DE LA TAILLE A, VIELLEFOND A, BERGER N, BOUCHER E, DE FROMONT M, FONDIMARE A, MOLINIE V, PIRON D, SIBONY M, STAROZ F, TRILLER M, PEL E, THIOUNN N, RUBIN MA.: Evaluation of the interobserver reproducibility of Gleason grade of prostastic adenocarcinoma using tissue microarrays. Hum Pathol 2003; 34: 444-449.

22. BORQUE A, SANZ G, ALLEPUZ C, PLAZA L, GIL P, RIOJA LA.: The use of neural networks and logistic regression analysis for predicting pathological stage in men undergoing radical prostatectomy: a population based study. J Urol 2001; 166: 16721678.

Dr. F. Algaba Arrea

Sección de Patología. Fundación Puigvert

C/ Cartagena, 340-350

08025 Barcelona

(Trabajo recibido el 2 septiembre 2003) 